\title{
Modification of Gauss-Chebyshev quadrature for modelling of crack growth in the field of residual stresses
}

\author{
A. N. Galybin ${ }^{1,2}$ \& S. M. Aizikovich ${ }^{3}$ \\ ${ }^{1}$ Wessex Institute of Technology, Southampton, UK \\ ${ }^{2}$ Institute of Physics of the Earth, RAS, Moscow, Russia \\ ${ }^{3}$ Rostov-on-Don Technical University, Russia
}

\begin{abstract}
A modification of the Gauss-Chebyshev quadrature for solving singular integral equations appearing in plane fracture problems is proposed. This modification is aimed at accurate modelling of the crack growth in non-homogeneous stress fields, which is the case that presents certain difficulties because the positions of collocation points on the crack vary with the crack length. In the proposed modification they are fixed, which extends the application of the well-established technique for oscillating or piecewise loads acting on the crack surfaces. In the latter case, despite lowering the degree of approximation, the proposed method provides better accuracy in calculations as confirmed by comparisons of numerical and analytic results for some examples.
\end{abstract}

Keywords: singular integral equations, quadrature, crack, residual stresses.

\section{Introduction}

Some natural and artificial materials exhibit considerable level of residual stresses. The latter can significantly change fracture characteristics of the material and affect crack propagation. In practice the residual stresses are rarely known as continues functions of spatial coordinates but they can routinely be measured at discrete points. It is, therefore, important to possess accurate numerical methods for the prediction of crack behaviour based on experimental data. This paper is aimed to adapt the Gauss-Chebyshev quadrature method for studying crack propagation in elastic media under combined load of external and internal non-homogeneous stresses. 
Quadrature of the Gauss type is often applied for solving singular integral equations, SIEs, appearing in plane elastic problem especially for bodies with cracks. It is believed that this approach had been originally developed by Multhopp in 1938 for the wing equation (see references in [1]) and became known in fracture mechanics after the paper by Erdogan and Gupta [2] published in 1972. The approach attracts attention of many researches due to simple programming and high accuracy in calculations of fracture characteristics. However there are some restrictions in the application of the method, for instance, discontinuities in applied loads or irregular crack paths cannot be directly handled by this method. For instance, Savruk [3] applied a modified scheme for the case of concentrated loads, otherwise the solution will be trivial because of the zero right hand side of the linear system obtained after discretization of SIE.

Modelling of the crack growth in non-homogeneous stress fields also presents certain difficulties because the positions of collocation points on the crack vary with the crack length. The essence of the method is that the choice of collocation points depends on the nodes of quadrature formulae, which provides highest degree of approximation. The positions of the nodes are also dependent on the contour. Therefore, after every finite step in quasistatic crack growth the positions of the collocation points are different from those at the previous steps. As a result due the right hand sides of a linear system algebraic equations obtained after the SIE discretisation (i.e., the loads acting at the crack surfaces) are always calculated at different collocation points. This is unimportant if the distributions of internal stresses are described by polynomial functions of spatial coordinates (because of convergence). However the original method produce large errors for discontinuous loads. For the discrete data (on residual stress measurements) the arrays of the right hand sides will be strongly dependant on interpolation used to calculate the loads at the collocation points. This may lead to the loss of accuracy in calculation of stress intensity factors and crack paths especially for curvilinear cracks.

The paper reports a modification of the Gauss-Chebyshev quadrature for solving SIEs. The SIE for curvilinear crack in the form presented in [3] is used in the analysis, although the results can be easily applied to any other forms of SIE or systems of SIEs appearing in fracture mechanics problem see survey [4].

\section{Singular integral equation for curvilinear cracks}

The SIE of the problem for curvilinear cracks in an elastic plane has the form [3]

$$
\frac{1}{2 \pi} \int_{\Gamma}\left\{\left[\frac{2}{t-t^{\prime}}+k_{1}\left(t, t^{\prime}\right)\right] Q(t) d t+k_{2}\left(t, t^{\prime}\right) \overline{Q(t) d t}\right\}=N\left(t^{\prime}\right)+i T\left(t^{\prime}\right), \quad t^{\prime} \in \Gamma
$$

Here $\Gamma$ is the contour that represents the union of all $K$ cracks $\Gamma_{k}(k=1, \ldots K)$ and other boundaries; the sought function $Q(t)$ is proportional to the density of the jump of the complex displacement vector, $u(t)+i v(t)$, across $\Gamma$, this function is unbounded at the crack ends; $N\left(t^{\prime}\right)+i T\left(t^{\prime}\right)$ is the complex stress vector acting on 
$\Gamma$. For the case of cracks in the infinite plane the regular kernels in (1) are given as follows

$$
k_{1}\left(t, t^{\prime}\right)=\frac{d}{d t^{\prime}} \ln \frac{t-t^{\prime}}{\bar{t}-\vec{t}^{\prime}}, \quad k_{2}\left(t, t^{\prime}\right)=-\frac{d}{d t^{\prime}} \frac{t-t^{\prime}}{\bar{t}-\vec{t}}
$$

For other configurations, e.g. cracks in a half plane or in a plane with a circular hole these kernels have more complex expressions, see [3] for details.

In order to provide a unique solution of the SIE the following condition of single valuedness of displacements should take place (for non-intersecting cracks)

$$
\int_{\Gamma_{k}} Q(t) d t=0, \quad k=1, \ldots K
$$

If some of the cracks intersect of bifurcate then the condition (3) should be modified by assuming single-valuedness of displacements after complete traverse of the contours formed by intersecting cracks.

Let us further consider the case of a single curvilinear crack in the complex plane, i.e. $\Gamma=\Gamma_{1}$. This case is sufficient to address the goal of this paper; more complex cases do not introduce essential difficulties. form

It is convenient to introduce the parameterisation of the crack in the following

$$
t=t(s)=\int_{0}^{s} e^{i \theta(r)} d r, \quad a \leq s \leq b
$$

where $\theta=\theta(s)$ is the angle between the tangent to the crack path and the positive direction of the $x$-axis, $a$ and $b$ are parameters associated with the crack length, $2 L=b-a$. Substitution of (4) into (1) leads to the following SIE

$$
\frac{1}{\pi} \int_{a}^{b}\left\{\left[\frac{1}{s-r}+k_{1}(s, r)\right] \widetilde{Q}(s)+k_{2}(s, r) \overline{\widetilde{Q}(s)}\right\} d s=P(r), \quad a<r<b
$$

where $\widetilde{Q}(s)=Q(t(s)), \quad P(r)=N(t(r))+i T(t(r))$ and regular kernels in $(5)$ assume the form

$$
\begin{aligned}
& k_{1}(s, r)=e^{i \theta(s)} e^{-i \theta(r)} \operatorname{Re}\left(\frac{e^{i \theta(r)}}{t(s)-t(r)}\right)-\frac{1}{s-r} \\
& k_{2}(s, r)=\frac{1}{2} \frac{e^{-i \theta(s)}}{\overline{t(s)}-\overline{t(r)}}\left(1-\frac{t(s)-t(r)}{\overline{t(s)}-\overline{t(r)}} e^{-2 i \theta(r)}\right)
\end{aligned}
$$

Condition (4) assume the form

$$
\int_{a}^{b} q(t(s)) e^{i \theta(t(s))} d s=0
$$

By linear substitution 
18 Boundary Elements and Other Mesh Reduction Methods XXXIII

$$
s=\frac{b-a}{2} \xi+\frac{b+a}{2}, \quad|\xi|<1 ; \quad r=\frac{b-a}{2} \eta+\frac{b+a}{2}, \quad|\eta|<1
$$

the problem consisting of SIE (5) and condition (7) assume the following form

$$
\begin{gathered}
\frac{1}{\pi} \int_{-1}^{1}\left\{\left[\frac{1}{\xi-\eta}+K_{1}(\xi, \eta)\right] q(\xi)+K_{2}(\xi, \eta) \overline{q(\xi)}\right\} \frac{d \xi}{\sqrt{1-\xi^{2}}}=p(\eta),|\eta|<1 \\
\int_{-1}^{1} \frac{q(\xi) e^{i \theta(\xi)}}{\sqrt{1-\xi^{2}}} d \xi=0
\end{gathered}
$$

Here $q(\xi)$ is a new sought function bounded on [-1,1], it is defined as follows

$$
q(\xi)=\widetilde{Q}\left(\frac{b-a}{2} \xi+\frac{b+a}{2}\right) \sqrt{1-\xi^{2}}
$$

Regular kernels are

$$
\begin{aligned}
& K_{1}(\xi, \eta)=\frac{b-a}{2} k_{1}\left(\frac{b-a}{2} \xi+\frac{b+a}{2}, \frac{b-a}{2} \eta+\frac{b+a}{2}\right) \\
& K_{2}(\xi, \eta)=\frac{b-a}{2} k_{2}\left(\frac{b-a}{2} \xi+\frac{b+a}{2}, \frac{b-a}{2} \eta+\frac{b+a}{2}\right)
\end{aligned}
$$

As soon as a solution for $\mathrm{q}(\xi)$ is found he stress intensity factors can be calculated as follows

$$
K_{I}^{ \pm}-i K_{I}^{ \pm}=q( \pm 1) \sqrt{\pi L}
$$

Superscripts " \pm " corresponds to the right (left) crack tip, i.e. when $s=a, s=b$ respectively.

\section{Numerical implementation}

\subsection{Discretization of SIE quadratures for singular and regular integrals}

The Gauss-Chebyshev quadratures for regular integrals have the form [3,5]

$$
\begin{gathered}
\frac{1}{\pi} \int_{-1}^{1} \frac{F_{1}(\xi)}{\sqrt{1-\xi^{2}}} d \xi=\frac{1}{n} \sum_{k=1}^{n} F_{1}\left(\xi_{k}\right), \quad \xi_{k}=\cos \frac{2 k-1}{2 n} \pi, \quad k=1 \ldots n \\
\frac{1}{\pi} \int_{-1}^{1} \sqrt{1-\eta^{2}} F_{2}(\eta) d \eta=\frac{1}{n} \sum_{j=1}^{n-1}\left(1-\eta_{j}^{2}\right) F_{2}\left(\eta_{j}\right), \quad \eta_{j}=\cos \frac{j \pi}{n}, \quad j=1 \ldots n-1
\end{gathered}
$$

Here $\xi_{k}$ and $\eta_{j}$ are zeroes of the Chebyshev polynomials of the first and second kind correspondingly

$$
\begin{gathered}
T_{n}(\eta)=\cos (n \arccos \eta) \\
U_{n-1}(\eta)=\cos (n \arccos \eta) / \sqrt{1-\eta^{2}}
\end{gathered}
$$

Formulas (14) and (15) are accurate for polynomials of (2n-1) and (2n-3) degrees correspondingly. 
Savruk [3] refers to the following quadrature for the singular integral

$$
\frac{1}{\pi} \int_{-1}^{1} \frac{1}{\sqrt{1-\xi^{2}}} \frac{q(\xi)}{\xi-\eta} d \xi=\frac{1}{n} \sum_{k=1}^{n} \frac{q\left(\xi_{k}\right)}{\xi_{k}-\eta}+\frac{q(\eta) U_{n-1}(\eta)}{T_{n}(\eta)}
$$

It can be shown that the following quadrature is also valid

$$
\frac{1}{\pi} \int_{-1}^{1} \sqrt{1-\eta^{2}} \frac{q(\eta)}{\eta-\xi} d \eta=\frac{1}{n} \sum_{j=1}^{n-1}\left(1-\eta_{j}^{2}\right) \frac{q\left(\eta_{j}\right)}{\eta_{j}-\xi}-\frac{q(\xi) T_{n}(\xi)}{U_{n-1}(\xi)}
$$

Quadratures (18) and (19) are accurate for polynomials of $2 n$ and (2n-2) degrees respectively.

Application of (14) and (18) to SIE (9) leads to the following functional equation

$$
\frac{1}{n} \sum_{k=1}^{n}\left\{\left[\frac{1}{\xi_{k}-\eta}+K_{1}\left(\xi_{k}, \eta\right)\right] q_{k}+K_{2}\left(\xi_{k}, \eta\right) \bar{q}_{k}\right\}+\frac{q(\eta) U_{n-1}(\eta)}{T_{n}(\eta)}=p(\eta), \quad|\eta|<1
$$

The condition of single valuedness assumes the form

$$
\sum_{k=1}^{n} q_{k} e^{i \theta\left(\xi_{k}\right)}=0
$$

Second terms in (18) and (19) formulas vanish if $\eta_{j}$ and $\xi_{k}$ are used as the collocation points correspondingly. In this case (20) assume its usual form for the numerical implementation and it is accurate for polynomials of $(2 n-1)$ degree.

\subsection{Quadrature with reduced accuracy}

Usual way in calculation of SIFs assumes extrapolation of the obtained solution up to the ends of the interval. This is based on the interpolation formulas, for instance, on the following one [3]

$$
q(\eta)=\frac{T_{n}(\eta)}{n} \sum_{k=1}^{n} \frac{(-1)^{k} \sqrt{1-\xi_{k}^{2}}}{\xi_{k}-\eta} q_{k}, \quad|\eta|<1
$$

The use of formulas like this always leads to the loss of accuracy because (22) is accurate for polynomials of $(n-1)$ degree while the solution obtained for $q_{k}$ is accurate for polynomials of $(2 n-1)$ degree.

Substitution of (22) into (18) leads to the following quadrature for the singular integral

$$
\frac{1}{\pi} \int_{-1}^{1} \frac{1}{\sqrt{1-\xi^{2}}} \frac{q(\xi)}{\xi-\eta} d \xi=\frac{1}{n} \sum_{k=1}^{n} \frac{1+(-1)^{k} U_{n-1}(\eta) \sqrt{1-\xi_{k}^{2}}}{\xi_{k}-\eta} q_{k}, \quad|\eta|<1
$$

Since interpolation is accurate for polynomials of $(n-1)$ degree, quadrature (23) is accurate for polynomials of the same degree if $\eta$ is an arbitrary point on [$1,1]$ (including the ends); at the points $\eta_{j}(23)$ is still accurate for polynomials of $2 n$ degrees because the additional term in the numerator vanishes. For regular kernels and condition of single valuedness, one can use (14) that is accurate for 
polynomials of $(2 n-1)$ degree. Therefore, the SIE is replaced by the following functional equation on the interval $|\eta|<1$

$$
\frac{1}{\pi} \sum_{k=1}^{n}\left\{\left[\frac{1+(-1)^{k} U_{k-1}(\eta) \sqrt{1-\xi_{k}^{2}}}{\xi_{k}-\eta}+K_{1}\left(\xi_{k}, \eta\right)\right] q_{k}+K_{2}\left(\xi_{k}, \eta\right) \bar{q}_{k}\right\}=p(\eta)
$$

which is accurate for polynomials of

- $\quad(n-1)$ degree for arbitrary $\eta$ and

- $\quad(2 n-1)$ degree if the roots of $\mathrm{U}_{n-1}(\eta)$ are used as collocation points (in this case (24) coincides with (20))

Let $\eta_{j}(j=1 \ldots J)$ be collocation points chosen on $[-1,1]$ such that $J \geq n-1$ then the following system of $(J+1)$ linear algebraic (complex) equations, SLAE, is obtained

$$
\sum_{k=1}^{n}\left(D_{k j} q_{k}+E_{k j} \bar{q}_{k}\right)=p_{j}, \quad \sum_{k=1}^{n} e^{i \theta\left(\xi_{k}\right)} q_{k}=0, \quad j=1 \ldots J
$$

Here the following notations have been introduced

$$
D_{k j}=\frac{1+(-1)^{k} U_{k-1}\left(\eta_{j}\right) \sqrt{1-\xi_{k}^{2}}}{\pi\left(\xi_{k}-\eta_{j}\right)}+\frac{1}{\pi} K_{1}\left(\xi_{k}, \eta_{j}\right), \quad E_{k j}=\frac{1}{\pi} K_{2}\left(\xi_{k}, \eta_{j}\right), p_{j}=p\left(\eta_{j}\right)
$$

Complex system (25) is further reduced to a real form by introducing the following array of $2 n$ real unknowns $X_{k}$

$$
q_{k}=X_{k}+i X_{n+k}, \quad k=1 \ldots n
$$

These unknowns can be placed in an array $\mathbf{X}$ and then (37) can be presented as

$$
\mathbf{A X}=\mathbf{B}
$$

Here $\mathbf{A}$ is $(2 J+2) \mathbf{x}(2 n)$ real matrix and $\mathbf{B}$ is $(2 J+2)$ real array.

Solution of the overspecified system (27) is obtained by the least squares method

$$
\mathbf{X}=\left(\mathbf{A}^{T} \mathbf{A}\right)^{-1} \mathbf{A}^{T} \mathbf{B}
$$

where superscript " $T$ " stands for the transposed matrix.

It should be mentioned that Kim [6] obtained a somewhat similar system for the case of simplest SIE, i.e. when $K_{1}(\xi, \eta)=K_{2}(\xi, \eta) \equiv 0$, However he has not acknowledged the fact that the application of Lagrange interpolation reduces the accuracy of discretization and restricted his consideration by the case $J=3 n-1$ in which $\eta_{j}$ are zeroes of $\mathrm{U}_{3 n-1}(\eta)$.

Despite the reduction in accuracy, SLAE (27) may provide better results for some cases of loading then the conventional approach (the latter follows directly from (24) by using ( $n-1)$ roots of $\mathrm{U}_{n-1}(\eta)$ as collocation points $\left.\eta_{j}\right)$ as, for instance, if continuous load oscillates sufficiently stronger then polynomials of $(2 n-1)$ degree or it is a piecewise function. In such cases the reduction of accuracy in conventional approach (due to incomplete account of the right hand side) can affect the results more significantly than the decrease of accuracy caused by the 
application of quadrature (24) to the left hand side of the SIE. An example supporting this statement is presented in the next section.

\section{Examples}

\subsection{Straight crack under oscillating load}

Stress intensity factors, SIFs, for a straight crack on the interval $(-L, L)$ subjected to normal load, $p=p(t)$ can be calculated exactly as follows

$$
K_{I, \text { ideal }}^{ \pm}=-\frac{1}{\sqrt{\pi L}} \int_{-L}^{L} \sqrt{\frac{L \pm t}{L \mp t}} p(t) d t
$$

An example of random tractions $p(t)$ is examined further on, see Fig. 1. Values of normal tractions have randomly been generated at 21 equidistant points (including the ends) as follows

$$
P=1,8,9,10,5,10,8,4,2,7,2,10,8,2,8,8,3,5,4,7,8 \text {. }
$$

After that the continuous tractions are found by either piecewise linear or cubic spline interpolation, which allows to calculate the ideal SIFs by (29) as follows

$K_{I \text { ideal }}^{+}=6.289$ and $K_{I \text { idea }}^{-}==6.090$ for cubic spline interpolation and

$K_{I \text { idea }}^{+}=6.262$ and $K_{I \text { idea }}^{-}=5.961$ for piecewise linear interpolation.

The following errors have been found for this particular example $(n=10)$ for cubic spline interpolation: $20.9 \%$ and $28.2 \%$ in the usual approach and $0.1 \%$ and $0.5 \%$ for the modified quadrature with $J=100$;

for piecewise linear interpolation: $20.4 \%$ and $28.2 \%$ in the usual approach and $0.2 \%$ and $0.1 \%$ for the modified quadrature with $J=100$.

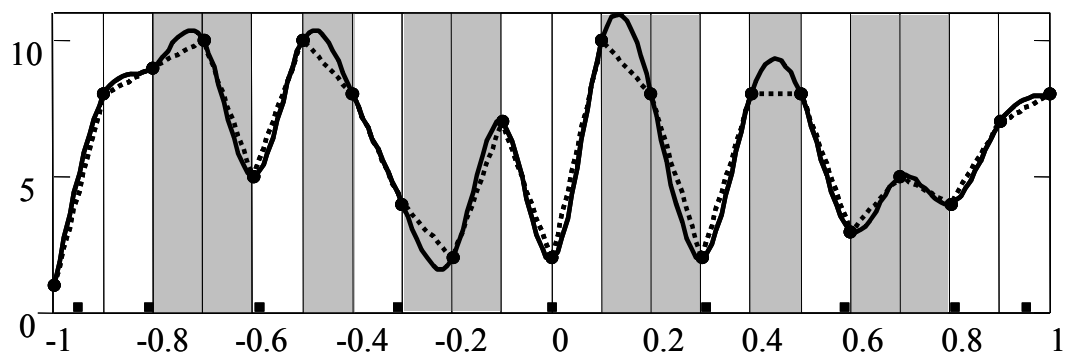

Figure 1: The load: circles are random data; solid line is cubic spline interpolation; dot line is linear interpolation; squares are collocation points, $\eta$; shaded areas represent the intervals not covered by the collocation points in the usual method.

These results demonstrate that the modified quadrature provides much better accuracy for the calculation of SIFs although it has less degree of accuracy than the usual scheme. This is explained by the positions of the collocation points in the usual approach. They are depicted by squares in Fig. 1 from which one can 
see that they do not cover a large part of the entire interval. For illustration, the whole interval is partitioned into 20 subintervals as shown in the Fig. 1 and then one can observe some intervals (shaded in the figure) that do not contain any collocation points. Since neither linear nor spline interpolations are polynomials of the $20^{\text {th }}$ degree (in which case one would have obtained accurate result for $n=10$ used in the example), the application of the usual approach leads to large errors because it is not able to address all the oscillations. In the meantime, the modified quadrature reflects all particularities of the load shown in the figure because 5 collocation points are placed on every subinterval if $J=100$.

\subsection{Straight crack under step-like loads}

The scheme of loading is presented in Fig. 2, where the uniform load is of unit intensity, $p=1$; it covers both symmetrical and asymmetrical loads. Ideal stress intensity factors are found from (29) as follows

$$
\frac{K_{I, \text { ideal }}^{ \pm}}{p \sqrt{\pi L}}=\arcsin \frac{R_{0}+\rho}{L}+\arcsin \frac{R_{0}-\rho}{L} \mp \sqrt{1-\left(\frac{R_{0}+\rho}{L}\right)^{2}} \pm \sqrt{1-\left(\frac{R_{0}-\rho}{L}\right)^{2}}
$$

where $\rho=0$ for symmetrical loading.

During calculations the number of collocations, $J$, varies such that at least one collocation point is placed between the neighbouring roots of $U_{n-1}(\eta)=0$. Then $J$ is found through the minimum distance between the neighbouring roots

$$
J=\beta\left[\min _{m=1 . . . n-2}\left(\cos \frac{\pi m}{n}-\cos \frac{\pi(m+1)}{n}\right)\right]^{-1}
$$

Here parameter $\beta$ determines the minimum number of collocation points on the smallest interval between $m$ and $m+1$ zeroes of $U_{n-1}(\eta)$.

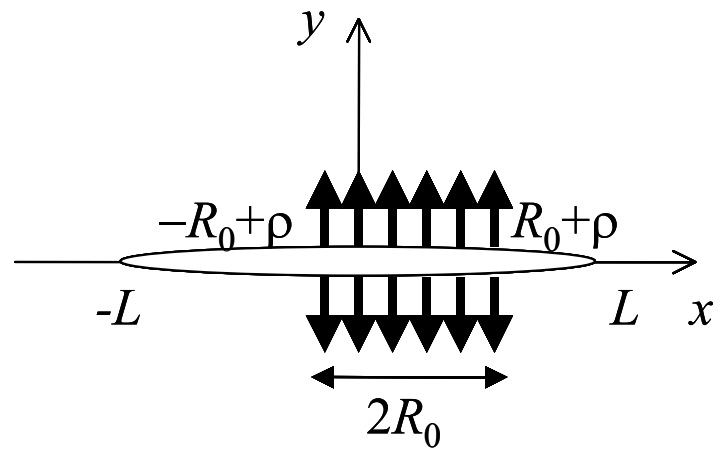

Figure 2: Cracks under step-like loads (symmetric (a) and (b) and asymmetric (c)).

It has been found that in order to obtain SIFs for crack loaded by a step-like load within $1 \%$ accuracy it is necessary to increase the number of either nodes, $n$, or/and collocation points, $J$. However, in the conventional scheme the accuracy 
varies depending on $n$ even for large $n>100$. When $n$ is fixed then the increase of $J$ refines SIFs. Some results of calculations are shown below.

Example 1. $R_{0}=0.7 \rho=0$ (load at the ends), $n=12$, error $32.7 \%$ for the usual approach. For the modified scheme:

$J=15(\beta=1)$ gives qualitatively incorrect result (negative SIF)

$J=45(\beta=3), 6.15 \%$ error

$J=149(\beta=10)$, error is $0.3 \%$, no further improvement

$J=445(\beta=30), 1.5 \%$ error (loss of accuracy)

Example 2. $R_{0}=0.7 \rho=0$ (load in the middle), $n=12$, error $32.5 \%$ for the usual approach. For the modified scheme:

$J=15(\beta=1), 146.6 \%$ error

$J=45(\beta=3), 6.3 \%$ error

$J=149(\beta=10)$ error is $0.3 \%$, no further improvement

$J=445(\beta=30), 1.5 \%$ error (loss of accuracy)

Example 3. Load on $(0.3,0.4)$. The right SIF is zero for $n=57$ (the left SIF has error of $3.1 \%$ ) in the usual scheme while errors of $0.4 \%$ have been found for the modified scheme.

These examples indicate that the usual approach is not reliable and should be rejected for the case of step-like load. The modified quadrature provides reasonable results. The value of $\beta=10$ can be recommended for all calculations, however the increase of approximation is necessary with the decrease of the area where the load is applied. The use of $n$ multiple of 4 can also be recommended for symmetrical cases (agrees with [7]), it gives minimum errors as compared with other close numbers.

\subsection{Approximation of curved cracks by polygonal lines}

For the case of circular crack depicted in Fig. 3 the exact expressions for SIFs are known, see, for instance, [3].

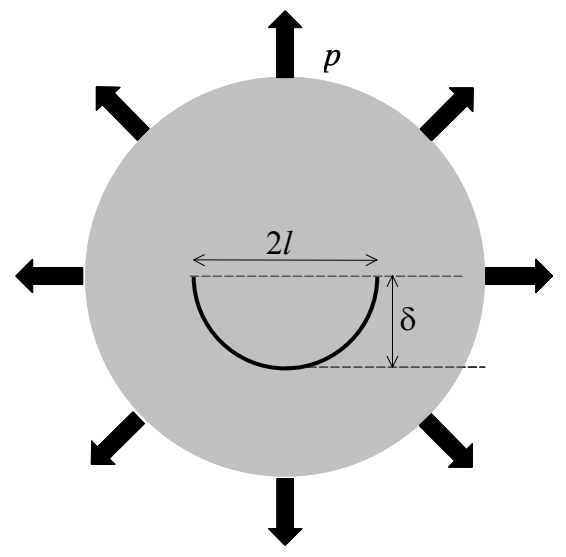

Figure 3: Circular crack in the plate subjected to biaxial uniform tension. 
Curvilinear cracks of complex geometry can be approximated by piecewise continuous contours of simple geometry like linear segments and circular arks or by using spline interpolations. We use linear approximation by polygonal lines that may introduce essential errors. However the results obtained demonstrate that the use of quadrature (24) does not induce additional loss of accuracy.

It has been assumed that the crack consists of $2 M$ linear segments of the length $\Delta s$ each. The results presented for the case when $\delta=l$ in which the ideal SIFs are $K_{\mathrm{I}}=K_{\mathrm{II}}=0.4714 l^{1 / 2}$. In all calculations $n=8,12,16,24$ and $\Delta \mathrm{s}=0.1$ and 0.05 .

For $\Delta s=0.1(M=16)$ maximum of errors in calculations of SIFs was greater than $3 \%$ regardless of $n$ and $J$. Thus, this approximation is not sufficient.

For $\Delta s=0.05(M=32)$ maximum of errors in calculations of SIFs was greater than $3 \%$ for $n=8$ regardless of $J$. For $n=12,16$ and 24 the maximum errors were $1 \%$ for the usual approach and for the modified scheme they were more than $3 \%$ for $J=2 M$; and $1-3 \%$ for $J=4 M, 10 \mathrm{M}$.

\section{Conclusions}

The main advantage of the proposed numerical scheme is a possibility to calculate the right hand side of the SLAE at the collocation points whose positions are fixed. The number of collocation points is selected considerably greater that the number of nodes, which leads to an overspecified system of SLAE. On one hand this results in reduction of accuracy as the original method is accurate for polynomials of $(2 n-1)$ degree while the modified method for polynomials of $(n-1)$ degree. On the other hand such accuracy is still better than in the traditional BEM methods, thus, the approach gives an effective way to analyse the crack growth by using the same datasets of the loads acting on the crack surfaces. This is important for modelling of crack propagation in the field of residual stresses or random stress fluctuations.

\section{References}

[1] Kalandiya, A.I. 1975. Mathematical methods of two-dimensional elasticity, Mir Publishers, Moscow.

[2] Erdogan, F. and Gupta, G.D. 1972. On the numerical solution of singular integral equations, Quart. Appl. Math. 29, 525-534.

[3] Savruk, M.P. 1981, 2D problems of elasticity for bodies with cracks, Naukova Dumka, Kiev.

[4] Chen, Y.Z. 1995. A survey of new integral equations in plane elasticity crack problem, Eng. Fract. Mech. 51, 97-134.

[5] Abramowitz, M. and Stegun, I.A. 1970. Handbook of mathematical functions. Dover, New York.

[6] Kim, S. 1999. Numerical solutions of Cauchy singular integral equations using generalized inverses, Computers Math. Applic. 38,183-195

[7] Kim, S. 1998. Solving singular integral equations using Gaussian quadrature and overdetermined system, Computers Math. Applic. 35, 63-71. 